Bull. Austral. Math. Soc.

VOL. 38 (1988) [171-176]

\title{
ON CONJUGACY CLASSES IN FINITE LOOPS
}

\author{
T. KEPKA AND M. NIEMENMAA
}

\begin{abstract}
The role of the conjugacy relation is certainly important in the structure theory of groups. Here we study this relation in a considerably more general setting, namely in the theory of loops. We first recall some basic facts about quasigroups, their multiplication groups, their inner mapping groups and the conjugacy relation. After this we estimate the size and the number of the conjugacy classes and we study the structure of loops having only two conjugacy classes. Finally, the values of the centraliser function are discussed.
\end{abstract}

\section{Preliminaries}

Let $Q$ be a finite loop (that is a finite groupoid with unique division and an identity element usually denoted by $e$ ). For every $a \in Q$, the left translation $L_{a}$ and the right translation $R_{a}$ are permutations on $Q$ defined by: $L_{a}(x)=a x$ and $R_{a}(x)=x a$ for every $x \in Q$. The set of all left and right translations generates a subgroup $M(Q)$ of $S(Q)$ (the group of all permutations on $Q$ ). We say that $M(Q)$ is the multiplication group of $Q$. Clearly, $M(Q)$ is transitive on $Q$ and the stabilisers of elements of $Q$ are conjugate in $M(Q)$. We denote by $I(Q)$ the stabiliser of the indentity element $e$; thus $I(Q)=\{P \in M(Q): P(e)=e\}$. This permutation group is called the inner mapping group of $Q$. Now card $(M(Q))=\operatorname{card}(Q) \cdot \operatorname{card}(I(Q))$.

Recall that the left, right and middle nucleus of $Q$ are defined as follows:

$$
\begin{aligned}
& N_{l}(Q)=\{a \in Q: a \cdot x y=a x \cdot y \text { for all } x, y \in Q\} \\
& N_{r}(Q)=\{a \in Q: x y \cdot a=x \cdot y a \text { for all } x, y \in Q\} \\
& N_{m}(Q)=\{a \in Q: x a \cdot y=x \cdot a y \text { for all } x, y \in Q\}
\end{aligned}
$$

Further, $N(Q)=N_{l}(Q) \cap N_{r}(Q) \cap N_{m}(Q)$ is called the nucleus of $Q$ and $C(Q)=\{a \in$ $\left.N(Q): L_{a}=R_{a}\right\}$ is the centre of $Q$. All the nuclei are subloops of $Q$ and the centre is an abelian subgroup. We characterise the centre by

LEMmA 1.1. $C(Q)=\{a \in Q: I(Q)(a)=a\}=\left\{a \in Q: L_{a} \in Z(M(Q))\right\}=$ $\left\{a \in Q: R_{a} \in Z(M(Q))\right\}$

For proof, see $[3$, pp. 60-62] and [8, pp. 217-218].

Received 28 October, 1987

Copyright Clearance Centre, Inc. Serial-fee code: 0004-9729/88 \$A2.00+0.00. 
From Lemma 1.1 it follows that the groups $C(Q)$ and $Z(M(Q))$ are isomorphic. It is also very easy to see that $I(Q)=\{1\}$ if and only if $Q$ is an abelian group.

Now $I(Q)$ is a permutation group on $Q$ and this means that its orbits determine an equivalence relation $\alpha$ on $Q$. If $(a, b) \in \alpha$, then we say that the elements $a$ and $b$ are conjugate in $Q$. For any $a \in Q, I(Q)(a)$ is the set of elements conjugate to a. By Lemma 1.1, every element of $C(Q)$ forms a one-element class of $\alpha$. Clearly, $\operatorname{card}(I(Q)(a))$ divides card $(I(Q))$ for every $a \in Q$.

Theorem 1.1. Suppose that $Q$ is not an abelian group. Then the factor loop $Q / C(Q)$ contains at least three elements.

Proof: Put $S=Q / C(Q)$. If $\operatorname{card}(S)=1$, then $Q=C(Q)$, a contradiction. Next assume that $\operatorname{card}(S)=2$. It follows that there is an element $a \in Q$ such that $Q=C(Q) \cup a C(Q), C(Q) \cap a C(Q)=\emptyset$. Let $x, y \in C(Q)$. Now $a x \cdot a y=$ $a^{2} \cdot x y, a(a x \cdot a y)=\left(a \cdot a^{2}\right)(x y)=\left(a^{2} \cdot a\right)(x y)=a^{2}(a \cdot x y)=a^{2}(a x \cdot y)=a^{2}(x a \cdot y)=$ $a^{2}(x \cdot a y)=a^{2} x \cdot a y=(a \cdot a x)(a y)$. We conclude that $a \in N_{l}(Q)$. In a similar way we can show that $a \in N_{r}(Q), a \in N_{m}(Q)$ and, finally, $a \in C(Q)$. But then $C(Q)=a C(Q)$, a contradiction. Thus $\operatorname{card}(S) \geqslant 3$.

Now let $n=\operatorname{card}(Q), r=$ the number of conjugacy classes and $m=\operatorname{card}(C(Q))$. We have

LEMMA 1.2. If $Q$ is an abelian group, then $m=r=n$. If $Q$ is not an abelian group, then $n \geqslant 5,2 \leqslant r \leqslant 2 n / 3$ and $1 \leqslant m \leqslant n / 3$.

Proof: If $n \in\{1,2,3,4\}$ then $Q$ is abelian group. If $Q$ is not an abelian group, then $n \geqslant 5$ and $r \geqslant 2$. By Theorem 1.1, $m \leqslant n / 3$ and since $r-m \leqslant(n-m) / 2$, it follows that $r \leqslant 2 n / 3$.

For basic facts about groups and loops we refer to [7] and [3].

\section{BOUNDS FOR THE NUMBER OF CONJUGACY CLASSES}

Let $Q$ be a loop of order $n$. A subset $M$ of $Q$ is said to be tame if there exists $P \in I(Q)(P \neq 1)$ Such that $P(x)=x$ for every $x \in M$. Now we define

$$
\begin{aligned}
& t(Q)=\max \{\operatorname{card}(M): M \text { is tame }\} \text { and } \\
& t(Q)=0, \quad \text { if } I(Q)=\{1\}
\end{aligned}
$$

We know already that $I(Q)=\{1\}$ if and only if $Q$ is an abelian group. If $Q$ is a nonabelian group, then $t(Q)=\max \left\{\operatorname{card}\left(C_{Q}(x)\right): x \in Q-Z(Q)\right\}$. This means that we are dealing with large centralisers and we shall return to this topic in section four. Now we shall use $t(Q)$ in order to estimate $m$ and $r$. 
LEMMA 2.1. If $Q$ is not an abelian group, then $t(Q) \geqslant 2 m$.

Proof: Suppose that $Q$ is not commutative. Then $L_{a} \neq R_{a}$ for some $a \in Q$ and $1 \neq P=R_{a}^{-1} L_{a} \in I(Q)$. Clearly, $a \notin C(Q)$ and $P(x)=x$ for every $x \in$ $C(Q) \cup a C(Q)$. It follows that $t(Q) \geqslant 2 m$.

Now we assume that $Q$ is commutative. Since $Q$ is not associative we can find a permutation $S$ such that $1 \neq S=L_{a}^{-1} L_{b}^{-1} L_{b a} \in I(Q)$. Here $b \notin C(Q)$ and for any $c \in C(Q)$ we have

$$
b a \cdot b c=(b a \cdot b) c=(b \cdot a b) c=b(a b \cdot c)=b(a \cdot b c),
$$

and so $S(b c)=b c$. But then $S(x)=x$ for every $x \in C(Q) \cup b C(Q)$ and again $t(Q) \geqslant 2 m$. The proof is complete.

Now assume that $Q$ is not an abelian group (and hence $n \geqslant 5$ ). Let $Q_{1}, \ldots, Q_{r}$ be the conjugacy classes and $n_{1}=\operatorname{card}\left(Q_{1}\right) \leqslant n_{2}=\operatorname{card}\left(Q_{2}\right) \leqslant \cdots \leqslant n_{r}=\operatorname{card}\left(Q_{r}\right)$. Then $1 \leqslant m<r, n_{1}=\cdots=n_{m}=1$ and $2 \leqslant n_{i}, i=m+1, \ldots, r$. We next establish

TheOREM 2.1. Let $m+1 \leqslant j \leqslant r$. Then $n \leqslant t(Q) n_{j}$.

Proof: Assume by way of contradiction that $n>t(Q) n_{j}$ for some $j(m+1 \leqslant$ $j \leqslant r)$. Now $P_{x}=R_{x}^{-1} L_{x} \in I(Q)$ for every $x \in Q$. We shall first prove that $P_{a}=1$ for every $a \in Q_{j}$. If $a \in Q_{j}$, then $P_{x}(a) \in Q_{j}$ for every $x \in Q$. For every $b \in Q_{j}$ we define the set

$$
A_{b}=\left\{x \in Q: P_{x}(a)=b\right\} .
$$

Now $n=\sum_{b \in Q_{j}} \operatorname{card}\left(A_{b}\right)>t(Q) n_{j}$, hence $\operatorname{card}\left(A_{b}\right)>t(Q)$ for some $b \in Q_{j}$. Clearly, $L_{b}^{-1} R_{a}(x)=x$ for every $x \in A_{b}$. Furthermore, if $c \in A_{b}$, then $S=L_{c}^{-1} L_{b}^{-1} R_{a} L_{c} \in$ $I(Q)$ and $S(x)=x$ for every $x \in L_{c}^{-1}\left(A_{b}\right)$. Since card $\left(L_{c}^{-1}\left(A_{b}\right)\right)>t(Q)$, we conclude that $S=1$ and thus $c y \cdot a=b \cdot c y$ for any $y \in Q$. But then $z a=b z$ for every $z \in Q$, $a=b$ and hence $P_{a}=1$.

Next we prove that $Q_{j} \subseteq N_{r}(Q)$. Let $a \in Q_{j}$ and $u \in Q$. For every $x \in Q$, $D_{x}=L_{u}^{-1} L_{x}^{-1} L_{x u} \in I(Q)$ and $D_{x}(a) \in Q_{j}$. For every $b \in Q_{j}$, let

$$
B_{b}=\left\{x \in Q: D_{x}(a)=b\right\}
$$

As in the first part of the proof we can again find $b \in Q_{j}$ such that $\operatorname{card}\left(B_{b}\right)>t(Q)$. Now $R_{u}^{-1} R_{a}^{-1} R_{u b}(x)=x$ for every $x \in B_{b}$ and if $c \in B_{b}$, then $L_{c}^{-1} R_{u}^{-1} R_{a}^{-1} R_{u b} L_{c}=1$. It follows that $c y \cdot u b=(c y \cdot u) a$ for every $y \in Q$. Thus $z \cdot u b=z u \cdot a$ for every $u$, $z \in Q$, and $a=b$, and consequently $a \in N_{r}(Q)$.

Likewise it follows that $Q_{j} \subseteq N_{l}(Q)$. But then $Q_{j} \subset C(Q), n_{j}=1$ and $j \leqslant m$, a contradiction. 
ThEOREM 2.2. The following inequalities hold:

(i) $t(Q) \geqslant(r-m) n /(n-m)$,

(ii) $t(Q) \geqslant(3 r-n) / 2$,

(iii) $t(Q) \geqslant r-m+1$.

Proof:

(i) This follows directly from Theorem 2.1 .

(ii) By Lemma 1.2, $m \leqslant n / 3$ and since $(r-m) /(n-m) \geqslant(r-n / 3) /$ $(n-n / 3)$, it follows by (i) that $t(Q) \geqslant(r-n / 3) n /(n-n / 3)=$ $(3 r-n) / 2$.

(iii) Now $(r-m) n /(n-m)=(r-m)(n-m+m) /(n-m)=r-m+k$, where $k>0$.

We shall illustrate the situation by some examples.

Example 2.1. For every $n \geqslant 5$ there exists a loop $Q$ of order $n$ such that $M(Q)=$ $S(Q)$ (see [5, Theorem 3.1.1.]). Now $m=1, r=2$ and $t(Q)=n-2$.

Example 2.2. For every $n \geqslant 6$ there exists a loop $Q$ of order $n$ such that $M(Q)=$ $A(Q)$ (the alternating group). Here $m=1, r=2$ and $t(Q)=n-3$ (for details, see $[\boldsymbol{\theta}])$.

Example 2.3. Consider a loop $Q$ with the following multiplication table:

$\begin{array}{llllll}1 & 2 & 3 & 4 & 5 & 6 \\ 2 & 1 & 4 & 3 & 6 & 5 \\ 3 & 4 & 5 & 6 & 2 & 1 \\ 4 & 3 & 6 & 5 & 1 & 2 \\ 5 & 6 & 1 & 2 & 3 & 4 \\ 6 & 5 & 2 & 1 & 4 & 3\end{array}$

Here $m=2, r=4$ and $t(Q)=4$. Furthermore, $I(Q)$ is isomorphic to Klein's four group. The factor loop $Q / C(Q)$ is a group of order three.

Remark. If $Q$ is a group of order $n$, then the number of conjugacy classes has a lower bound, namely $r \geqslant \log (\log n) / \log 2$ (see [9]).

\section{LoOps with $t(Q)=2$}

Let $Q$ be a loop of order $n$ which is not an abelian group (hence $n \geqslant 5$ ). In this section we also assume that $t(Q)=2$. By Theorem 2.2 (iii), $r \leqslant t(Q)+m-1=m+1$ and obviously $r=m+1$. By Lemma $2.1,2=t(Q) \geqslant 2 m \geqslant m+1=r$ which gives $r=2$ and $m=1$. 
Now we can consider $I(Q)$ as a permutation group on $E=Q-\{e\}$. Clearly, $I(Q)$ is transitive on $E$ and $M(Q)$ is 2-transitive on $Q$. Furthermore, $I(Q)$ is a Frobenius group and therefore card $(I(Q))=k(n-1)$, where $k$ divides $n-2$.

THEOREM 3.1. If $t(Q)=2$, then $Q$ is simple and $Q$ is either commutative or anticommutative (that is $a b \neq b a$ whenever $e \neq a \neq b \neq e$ ).

Proof: Since $M(Q)$ is 2-transitive it is primitive and it follows that $Q$ is simple (see [10, p. 10]).

Suppose then that $a b=b a$ for some $e \neq a \neq b \neq e$. Put $P=R_{a}^{-1} L_{a}$, then $P(e)=e, P(a)=a$ and $P(b)=b$. Since $t(Q)=2$, we conclude that $P=1$. Consequently, $a x=x a$ for every $x \in Q$. Hence, if $S=R_{x}^{-1} L_{x}(x \neq e, x \neq a)$, then $S(e)=e, S(a)=a$ and $S(x)=x$ and necessarily $S=1$. But then $x y=y x$ for every $x, y \in Q$.

THEOREM 3.2. Let $Q$ be a loop which is not an abelian group and let $t(Q)=2$. Further, let $Q$ be of the smallest possible order. Then:

(i) if $A$ is a subloop of $Q(1 \neq A \neq Q)$, then $A$ is a group of order two;

(ii) $n \geqslant 6$.

\section{Proof:}

(i) If $A$ is not an abelian group then $A$ satisfies $t(A)=2$, too. This is a contradiction, hence $A$ is an abelian group. Assume now that card $(A) \geqslant 3$. By Theorem 3.1, $Q$ is commutative. Consider a permutation $P=L_{b}^{-1} L_{a}^{-1} L_{a b}$, where $a, b \in A$. It is easy to see that, in fact, $P=1$ and thus $a \cdot b x=a b \cdot x$ for every $x \in Q$. Similarly, if $S=R_{b}^{-1} R_{x}^{-1} R_{b x}$, where $b \in A$ and $x \in Q$, we conclude that $S=1$. Thus, $y \cdot b x=y b \cdot x$ for every $x, y \in Q$. Finally, if we put $U=R_{x}^{-1} L_{y}^{-1} R_{x} L_{y}$, then $U=1$ and we have shown that $y \cdot z x=y z \cdot x$ for every $x, y, z \in Q$. But then $Q$ is an abelian group, a contradiction.

(ii) Let $n=5$. Now $\operatorname{card}(I(Q))$ is either $=4$ or $=12$. Since $t(Q)=2$, it follows that $\operatorname{card}(I(Q))=12$, and so $\operatorname{card}(M(Q))=60$. This means that $M(Q)$ is the alternating group on $Q$. Now the complete list of non-associative loops of order five (see for example [5]) reveals the fact that any such loop generates at least one odd permutation, a contradiction.

It seems to be an open problem (at least to the authors) whether there exist loops $Q$ satisfying $t(Q)=2$.

\section{THE CENTRALISER FUNCTION}

For $n \geqslant 5$, we define the centraliser function $T$ by $T(n)=\min \{t(Q)$ : $Q$ is a loop of order $n$ which is not an abelian group . Similarly, for $n \geqslant 1$, put $t(n)=$ 
$\min \{t(G): G$ is a nonabelian group of order $n\}$ and $t(n)=0$ if all groups of order $n$ are abelian. Clearly, $T(n) \geqslant 2$ and $T(n) \leqslant t(n)$ provided $t(n) \neq 0$. Further $T(5)=3$ and $t(n)=0$ if and only if $n$ is cube-free and $(n, \varphi(n))=1$; we denote by $\mathcal{A}$ the set of all such integers $n$.

While nothing is known about $T(n)$ we are able to give a lower bound for $t(n)$. Recall that $t(G)=\max \left\{\operatorname{card}\left(C_{G}(x)\right): x \in G-Z(G)\right\}$.

Theorem 4.1. Let $n \geqslant 6, n \notin \mathcal{A}$. Then $t(n)>n^{1 / 4}$. Moreover, $t(n)>n^{1 / 2}$ provided that $n$ is odd.

Proof: Let $G$ be a nonabelian group of orcler $n$. If $G$ is soluble, then $t(G)>n^{1 / 2}$ (see [4]), hence $t(n)>n^{1 / 2}$ for $n$ odd. Thus we may assume that $G$ is not soluble, hence (by the odd order theorem) $n$ is even. If $Z(G / Z(G)) \neq\{1\}$, then $t(G)>n^{1 / 2}$ (see [1, Lemma 2]). If $Z(G)=\{1\}$, then we can use the theorem by Brauer and Fowler (see $[2]$ ) and $t(G)>n^{1 / 3}$. Finally, we assume that $Z(G) \neq\{1\}$ and $Z(G / Z(G))=\{1\}$. Now $H=G / Z(G)$ is of even order $k$ and hence we have an element $a Z(G) \in H$ such that $\operatorname{card}\left(C_{H}(a Z(G))\right)>k^{1 / 3}$ and also $\operatorname{card}\left(C_{G}(a)\right)>k^{1 / 3}$ (see [1, Lemma 1]). It follows that $t(G)>\max \left\{k^{1 / 3}, n / k\right\}$, hence $t(G)>n^{1 / 4}$. We conclude that $t(n)>n^{1 / 4}$. This completes the proof.

\section{REFERENCES}

[1] E.A. Bertrall, 'La'arge centralizers in finite soluble groups', Israel J. Math. 47 (1984), 335-344.

[2] R. Brauer and K. Fowler, 'On groups of even order', Ann. Math. 62 (1955), 565-583.

[3] R. Bruck, A Survey of Binary Systems (Springer-Verlag, Berlin-Heidelberg-New York, 1971).

[4] J. Cossey, 'Finite soluble groups have large centralizers', Bull. Austral. Math. Soc. 35 (1987), 291-298.

[5] J. Dénes and A.D. Keedwell, Latin Squares and Their Applications (Akadémiai Kiadó, Budapest, 1976).

[6] A. Drápal and T. Kepka, 'Alternating groups and latin squares' (to appear).

[7] B. Huppert, Endliche Gruppen I (Springer-Verlag, Berlin-Heidelberg-New York, 1967).

[8] T. Ilringer, 'Quasigroups, loops and centralizer rings', in Contributions to General Algebra 3.: Proceedings of the Vienna Conference, pp. 211-224, 1984.

[9] M. Newman, 'A bound for the number of conjugacy classes in a group', J. London Math. Soc. 43 (1968), 108-110.

[10] J.D.H. Smith, Multiplication Groups of Quasigroups, Preprint 603, Darmstadt, 1981.

Dr T. Kepka

Clliarles University

Prague

Cizechoslovakia
Dr M. Niemenmaa

Department of Mathematics

University of Oulu

Linnammaa

90570 Oulu

Finland 